\title{
Precursors for CVD growth of nanocrystalline diamond
}

\author{
(C) T. Soga, T. Sharda*, T. Jimbo \\ Department of Environmental Technology and Urban Planning, Nagoya Institute of Technology, \\ Nagoya 466-8555, Japan \\ * Research and Development, Seki Technotron Corp., \\ Tokyo 135-0042, Japan \\ E-mail: soga@elcom.nitech.ac.jp
}

\begin{abstract}
Variuos routes to grow nanocrystalline diamond films by chemical vapor deposition technique are reviewed. Among various routes, NCD films deposited on mirror polished silicon substrates by biased enhanced growth by microwave plasma chemical vapor deposition are described in detail. Qualitative concentration of NCD was assessed by Raman spectroscopy and $X$-ray diffraction patterns of the films. The hardness of the films approaches to that of natural diamond at optimized conditions while still having low amount of stress $(<1 \mathrm{GPa})$.
\end{abstract}

\section{Introduction}

Conventional polycrystalline diamond films composed of micrometer size of diamond crystal (microcrystalline diamond - MCD) grown by chemical vapor deposition (CVD) have surface roughness that limits its uses in many of the potential areas. For example, the high surface roughness is a major problem for machining and wear applications [1,2]. Two solutions can be suggested to overcome the problem of high surface roughness, either post-polishing can be adopted or naturally grown smooth films can be developed without compromising much with their useful properties. However, post-polishing is expensive and time consuming [3-5] and it may be considered to be better to concentrate on as-grown smooth and hard films [4-7]. Nanocrystalline diamond (NCD) films, that have superb tribological properties, can be a better alternative of post-polished conventional chemical vapor deposited diamond films. Moreover, smooth NCD films will be used as DNA chip, electrochemical electrode, heat sink, SAW filter, MEMS, NEMS, etc. Some studies (growth method properties and applications) that have been carried out on NCD in various laboratory all over the world can be found in detail in the most recent review article [8].

A common feature of the majority of the deposition techniques of CVD diamond films is a high concentration of hydrogen gas $\left(\mathrm{H}_{2}\right)$ used as one of the constituents with some hydrocarbon gas such as $\mathrm{CH}_{4}$. The high concentration of $\mathrm{H}_{2}$ results in the generation of large flux of atomic hydrogen, which is generally believed to play a central role in the various diamond CVD processes. The growth of diamond is described to take place mostly via surface processes of addition and abstaction of radicals from the gas-phase $[9,10]$. Before realizing the importance of growing naturally smooth surface, the aim in the area of CVD diamond was to maximize the crystalline quality of CVD diamond. However, diamond grown under non-optimum conditions, such as lower hydrogen concentration or higher carbon activity in the plasma, gives films with small grain size, e.g., several nanometers.

In the already existing ways to grow smooth NCD films, a number of techniques and conditions have been employed. As the need of having as-grown smooth diamond films have been realised in the last few years, new routes have also emerged that mostly aim to grow smooth NCD films. In this paper, based on the literature, some of the growth routes of NCD are briefly discussed followed by description on some properties of NCD film grown particularly using biased enhanced growth.

\section{Nanocrystalline diamond growth routes}

2.1. Hydrogen deficient gas-phase. One such process that has been studied in detales was developed at Argonne National Laboratory, USA $[11,12]$. In this process, carbon dimer $\left(\mathrm{C}_{2}\right)$ is used as a reactive species in hydrogen deficient $\left(\mathrm{CH}_{4} / \mathrm{Ar}\right.$ or $\left.\mathrm{C}_{60} / \mathrm{Ar}\right)$ microwave plasma CVD [11]. $\mathrm{C}_{2}$ is produced by replacing molecular hydrogen by argon and using $\mathrm{CH}_{4}$ or $\mathrm{C}_{60}$ as precursor gases and also by using $\mathrm{N}_{2} / \mathrm{CH}_{4}$ as the reactant gases in a microwave plasma CVD (MPCVD) system. The NCD films grown on diamond seeded substrates by this technique are composed of $3-15 \mathrm{~nm}$ diamond crystallites with up to $1-10 \% \mathrm{sp}^{2}$ carbon residing at the boundaries [10]. Lin et al. [13] studied the $\mathrm{CH}_{4} / \mathrm{H}_{2} / \mathrm{Ar}$ process in hot filament CVD (HFCVD) system and proposed a compositional map, which demarcates regions for a well-faceted diamond growth, NCD and nondiamond deposition. Well-faceted MCD $(2-10 \mu \mathrm{m})$ grow up to $90 \%$ Ar. They reported change in microstructure from MCD to NCD, with a grain size smaller than $50 \mathrm{~nm}$, at 95.5\% Ar addition.

The effects of addition of nitrogen to $\mathrm{CH}_{4} / \mathrm{H}_{2}$ have also been reported. Wu et al. [14,15] grew NCD films using $\mathrm{N}_{2} / \mathrm{CH}_{4} / \mathrm{H}_{2}$ in MPCVD system. NCD films with grain size of $8 \mathrm{~nm}$ embedded in $a-\mathrm{C}$ matrix was obtained without any hydrogen. The diamond crystallite size in their films increases from 20 to $50 \mathrm{~nm}$ while increasing hydrogen from 5 to $10 \mathrm{sccm}$, respectively.

Lee et al. $[16,17]$ developed a low temperatures process $\left(350<T<500^{\circ} \mathrm{C}\right)$ for low power but high growth rate (up to $2.5 \mu \mathrm{m} / \mathrm{h}$ ) NCD film by MPCVD. They used CO-rich $\mathrm{CO} / \mathrm{H}_{2}$ mixtures and obtained smooth NCD films consisting of $30-40 \mathrm{~nm}$ grain size. The temperature at which the peak growth rate is obtained in this temperature window 
decreases with increasing the $\mathrm{CO} / \mathrm{H}_{2}$ ratio. Recently, Teii et al. [18] also reported growth of NCD films of size $20 \mathrm{~nm}$ at low pressure ( $80 \mathrm{mTorr}$ ) at $700^{\circ} \mathrm{C}$ by inductively coupled plasma employing $\mathrm{CO} / \mathrm{CH}_{4} / \mathrm{H}_{2}$ and $\mathrm{O}_{2} / \mathrm{CH}_{4} / \mathrm{H}_{2}$. In this case a positive biasing of $20 \mathrm{~V}$ vas applied to the substrate to reduce the influence of ion bombardment. The films consisted of ball shape grains of size $100 \mathrm{~nm}$, which are further composed of $20 \mathrm{~nm}$ NCD grains.

2.2. Bias enhanced nucleation/growth using $\mathrm{CH}_{4} / \mathrm{H}_{2}$ gas system. In the case of the growth route for NCD by hydrogen deficient plasmas (section 2.1), mostly the substrates were pretreated externally before deposition either ultrasonically or mechanically using diamond or other abrasive powders. There is another well-established method to nucleate diamond internally in the conventional growth of MCD films called biased enhanced nucleation (BEN) [19] in which the substrates are biased negatively hydrocarbonrich mixture of hydrocarbon-hydrogen precursor gases. This method, which results in a high density of diamond nucleation $\left(10^{10} \mathrm{~cm}^{-2}\right.$ or more $)$, is the first step followed by another step or two steps to grow heteroepitaxial diamond films. In order to achieve overgrowth of diamond on these nuclei, the BEN process is followed by conventional growth in which the bias is put off and the growth is continued with lower hydrocarbon percentage in the gas-phase. Is was observed that in the later stages of growth, when the biasing is put off and hydrocarbon to hydrogen ratio is reduced, only the stable part of the nuclei continue to grow while remaining get etched off in the process due to increased concentration of hydrogen in the gas-phase [20].

To obtain an NCD film, Sharda et al. [21-23] suggested to achieve higher densities of diamond nucleation, similar to the BEN process, and, second, in the later stages to maintain the same high density and to continue their growth throughout the process. In order to materialize this idea, they extended the BEN in an MPCVD system for the whole growth process and termed their process biased enhanced growth (BEG) in which they obtained diamond nucleation and growth on silicon substrates in a single process. The details of the BEG process will be described later.

2.3. Diamond seeding. Yang et al. [24] grew transparent diamond films with a crystallite size below $70 \mathrm{~nm}$ in hydrogen and methane microwave plasma CVD on quartz substrates, ultrasonically pretreated by $0.5 \mu \mathrm{m}$ diamond powder, for $30 \mathrm{~min}$. Grain size and surface roughness were observed to decrease with increasing methane concentration. A significant reduction in grain size seems to occur at 3\% methane, which further reduces at 4\% methane though the nanodiamond concentration in the films also goes up. The crystallite size, as estimated by TEM, near the interface was $30 \mathrm{~nm}$ and increases to $65 \mathrm{~nm}$ near the growth side.

In another method, a diamond layer can be coated on substrates by dielectrophoresis or spraying method. Zhu et al. [25] attached thin nano-structured diamond films to silicon substrates by spraying or brushing technique using commercially available, produced by explosives, 10 to $100 \mathrm{~nm}$ size micro-polycrystalline diamond particles with each of the particle consisting of 1 to $20 \mathrm{~nm}$ crystallites and associated grain boundaries in aqueous suspension. Gohl et al. [26] deposited nanodiamond powder coating by dielectrophoresis technique. They used the nanodiamond powder of size 1-10 nm produced from the shock synthesis on a Si tip array, rough Si stumps and flat standard mirrorpolished $\mathrm{Si}$ substrates. $\mathrm{Xu}$ et al. [27] deposited nanostructured diamond coating on etched silicon substrates by dielectrophoresis method using $\sim 5 \mathrm{~nm}$ nanodiamond powder, produced by explosives, by suspending them in ethanol. Maillard-Schaller et al. [28] deposited 4-5 nm size diamond nanoparticles on flat $\mathrm{Si}(100)$ substrates by electrophoresis/dielectrophoresis. Hiraki [29] grew the diamond films at $200^{\circ} \mathrm{C}$ by nanodiamond seeding.

\section{Biased enhanced growth of NCD films}

In this section, we will discuss the growth of NCD by the BEG process in detail. The NCD films were grown by the BEG process in a $2.45 \mathrm{GHz}$ Seki Technotron Corporation, Japan (formerly Applied Science and Technology, USA) made the MPCVD system. The mirror-polished $\mathrm{Si}(100)$ substrates were kept on a Mo holder that rests on a graphite susceptor. No diamond powder of any other ex situ treatment was performed prior to the depositions. The substrate assembly was immersed in 5\% methane and hydrogen plasma. In a special arrangment to the substrate assembly, a quartz shield was used to cover the conducting parts of the subtrate holder assembly (other than the substrate). This assembly enhances the bias current density when a negative bias is applied to the substrate at low microwave powers without affecting the microwave plasma. The whole growth was performed for $60 \mathrm{~min}$ in a single stage run without breaking the bias to the substrate, unlike the conventional two or three stages process for the heteroepitaxial growth of diamond [30,31]. Applied biasing voltage was varied from 200 to $320 \mathrm{~V}$ while keeping other parameters constant. Films were grown at a pressure of 30 Torr $(\sim 4000 \mathrm{~Pa})$ with a microwave power of $1000 \mathrm{~W}$ at $600^{\circ} \mathrm{C}$. The substrate temperature was measured using a thermo-couple at the bachside of the substrate holder.

Structural characterizations of the films were carried out using Raman spectroscopy, $X$-ray diffraction (XRD), scanning electron microscopy (SEM) and atomic force microscopy (AFM). The laser Raman spectra were obtained in the range $1000-1700 \mathrm{~cm}^{-1}$ with a step of $1 \mathrm{~cm}^{-1}$. An $\operatorname{Ar}^{+}$-laser $(\lambda=488 \mathrm{~nm})$ of $200 \mu \mathrm{m}$ diameter spot size was used for recording the spectra. Hardness of the films was measured by nano-indentor (UMIS-2000) using a Berkovich diamond pyramid.

The stress in the films was calculated by measuring the radius of curvature of the substrates before and after the deposition using a modified Stoney's equation. The curvature of the films was measured by the Alpha-500 profiliometer. The length of a scanned sample segment was $5 \mathrm{~mm}$. 


\section{Properties of NCD films grown by biased enhanced growth}

A high resolution SEM photograph of the NCD film grown at $-200 \mathrm{~V}$ is shown in Fig. 1. Careful observation of the micrograph reveals that the film consists of bunches of sharp faceted nanocrystallites of a size less than $30 \mathrm{~nm}$. The transmission electron microscopic examination of the same film showed nanocrystallites confined in the form of oriented nanodiamond tubes of a size of $10-30 \mathrm{~nm}$ and a height of a few hundreds of $\mathrm{nm}$. Although, this structure resembles the columnar or dendritic kind of growth, nanocrystals confining in nanodiamond tubes is a unique feature of this new approach and method presented in this article. These nanodiamond tubes, having a high packing density of crystallites, appeared to be nearly parallel to the film growth direction. The corresponding electron diffraction patterns were indexed to diamond with the [111] and [220] diamond textured rings having their texture maxima parallel, respectively, to $[111]$ and $[220] \mathrm{Si}$ diffraction spots when superimposed. This indicated that majority of the NCD crystallites are preferentially oriented to the $\mathrm{Si}$ substrate. On the other hand, the film grown at $-320 \mathrm{~V}, 5 \% \mathrm{CH}_{4} / \mathrm{H}_{2}$ and $600^{\circ} \mathrm{C}$ substrate temperature showed a very different microstructure. This film also had a high packing density, however, of randomly oriented diamond crystallites of size $2-5 \mathrm{~nm}$. The detailed structure of the NCD film will be published elsewhere [32].

Fig. 2 shows the typical Raman spectra of the nanocrystalline films and conventional polycrystalline diamond film. The most significant feature in the Raman spectrum of the NCD film is the intense peak near $1150 \mathrm{~cm}^{-1}$ without any feature near $1332 \mathrm{~cm}^{-1}$, an unambiguous signature of cubic crystalline diamond. Although the origin of this peak is assigned to the trans-polyacetylene [33], it is widely accepted to be related to NCD [34]. Absence of any peak near $1332 \mathrm{~cm}^{-1}$, in spite of having an intense NCD feature, could be due to high density of defects incorporated in the

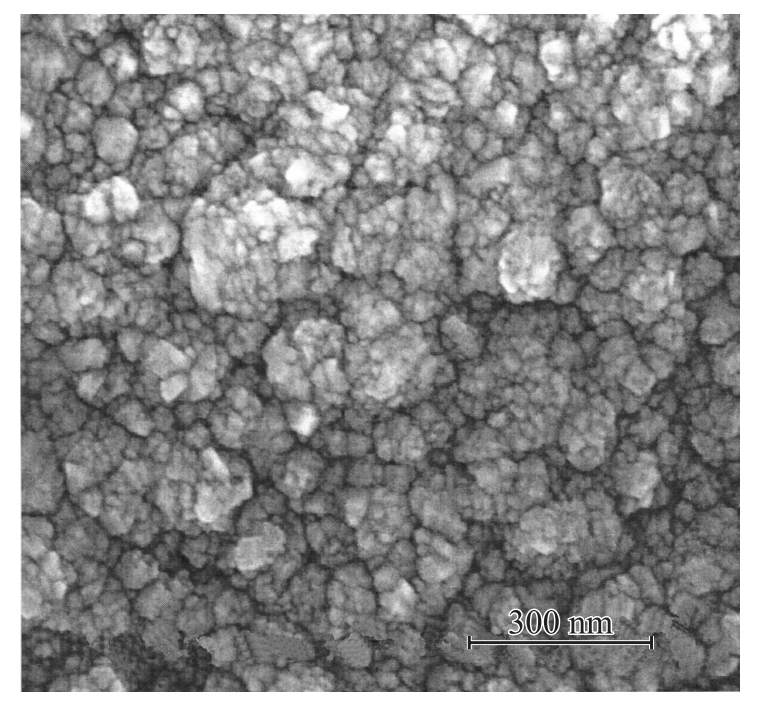

Figure 1. A high resolution SEM micrograph of an NCD sample.

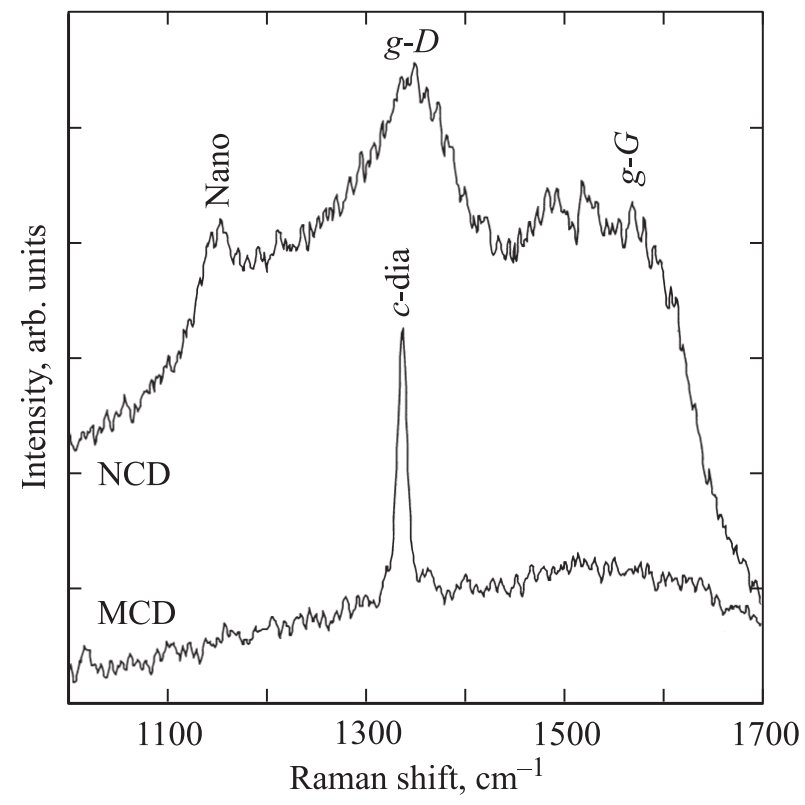

Figure 2. Typical Raman spectra of NCD film and MCD film.

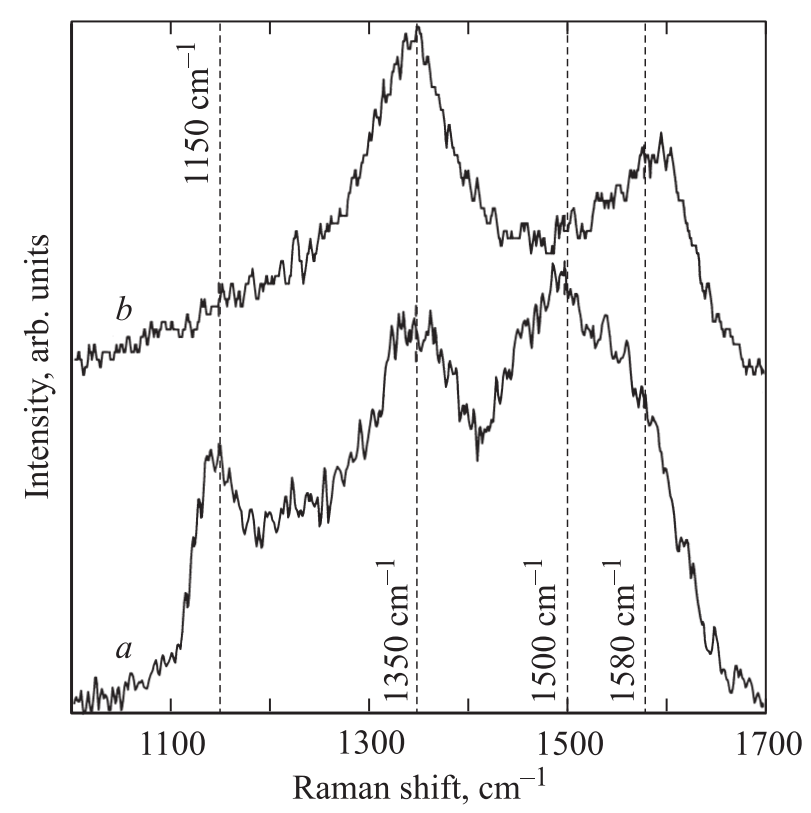

Figure 3. Raman spectra of NCD films at the bias voltage of $200(a)$ and $320 \mathrm{~V}(b)$.

films but could also be a sign of uniformly distributed shortrange $s p^{3}$ crystallites in the films [17,35]. Other significant bands near 1350 and $1580 \mathrm{~cm}^{-1}$ are well-known graphitic $D$ and $G$ bands. The Raman feature near $1500 \mathrm{~cm}^{-1}$ may be related to the disordered $s p^{3}$ carbon in the films [34]. The intensity of this band increases proportionally with the intensity of the NCD feature in the films as observed by others also [35]. High density of defects and a significant amount of graphitic carbon are, in fact, expected in the growth of uniformly distributed short range nanocrystals of diamond because of their large grain boundary area. 


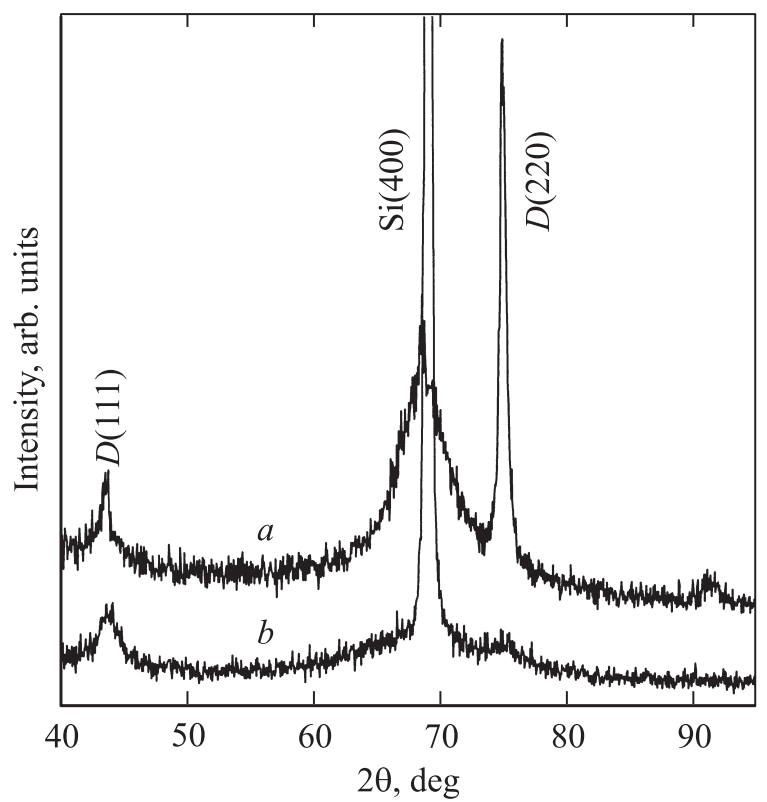

Figure 4. XRD patterns of the films deposited at the bias voltage of $200(a)$ and $320 \mathrm{~V}(b)$.

Fig. 3 shows the Raman spectra of the films deposited on $\mathrm{Si}(100)$ at the biasing voltages of 200 and $320 \mathrm{~V}$. It should be noted that the deposition didn't take place when the biasing voltage was lower than $200 \mathrm{~V}$. As can be seen, the intensity of the NCD Raman feature has almost vanished in the films grown at $320 \mathrm{~V}$. Moreover, there is a drastic variation in the position of the graphitic $G$ band in the film grown at $320 \mathrm{~V}$. It appears that relative concentration of $s p^{3}$ to $s p^{2}$ carbon of the films decreases with biasing voltage in the films. It suggests that the relative intensity of the NCD Raman feature decreased with increasing the biasing voltage. In fact, the NCD Raman feature appeared to have almost vanished in the films grown at $320 \mathrm{~V}$. Moreover, there was a drastic variation in the position of the graphitic $G$ band in the films grown at $320 \mathrm{~V}$. Overall, it appears that the relative concentration of $s p^{3}$ to $s p^{2}$ carbon of the films decreases with biasing voltage in the films. But it should be noted that no peaks associated with graphite could be identified in our films.

XRD patterns of the films at the biasing voltages of 200 and $320 \mathrm{~V}$ are shown in Fig. 4. The calculated interplanar spacing corresponding to the peaks at $2 \theta \sim 44.05$, $75.25 \pm 0.20^{\circ}$ in the XRD patterns of the films match closely with the inter-planar $d$-values of (111) and (220) planes of cubic diamond, respectively. It should be noted that the full width at half maximum (FWHM) of the diamond peaks in the films is in general high as compared to the MCD films. This is well correlated with the fact that diamond nanocrystallites are present in our films. It should be noted that no peaks associated with graphite or features related with amorphous carbon could be identified in our films.

Fig. 5 illustrates the summary of the properties grown at different biasing voltages. The hardness of the films decreases with increase in biasing voltage. Although not shown here, the hardness of NCD films grown by the BEG process at $200 \mathrm{~V}$ approaches to the hardness of natural diamond when the thickness increases to $4 \mu \mathrm{m}$ [22]. The Raman peak ratio $I_{n} / I_{g}$ (the ratio of $1150 \mathrm{~cm}^{-1}$ feature to graphite $G$ band) varies in the same fashion as the hardness of the films indicating that the relative concentration of NCD in the films may be responsible for the hardness of the films. The stress in the films increases with increasing bias voltage and increases drastically in the film grown at $320 \mathrm{~V}$. The samples grown at $320 \mathrm{~V}$ were visibly bent, and accounted for an enormous stress in the films [36]. It should be mentioned that no bending was observed in a sample, in a separate experiment, treated at bias voltage of $-320 \mathrm{~V}$ for an hour while using only hydrogen, i. e., without any methane in the gas-phase. This means that the bending occurs solely due to stress generating in the carbon film depositing on the wafers. To the best of our knowledge, there is no report of such an enormous amount of stress in carbon films. It may be mainly because the films, deposited by other groups, if having compressive stress more than $2 \mathrm{GPa}$, delaminated soon after the deposition due to weak adhesion [37,38]. However, in our case, a strong adhesion of the films to the substrate is developed making it possible to
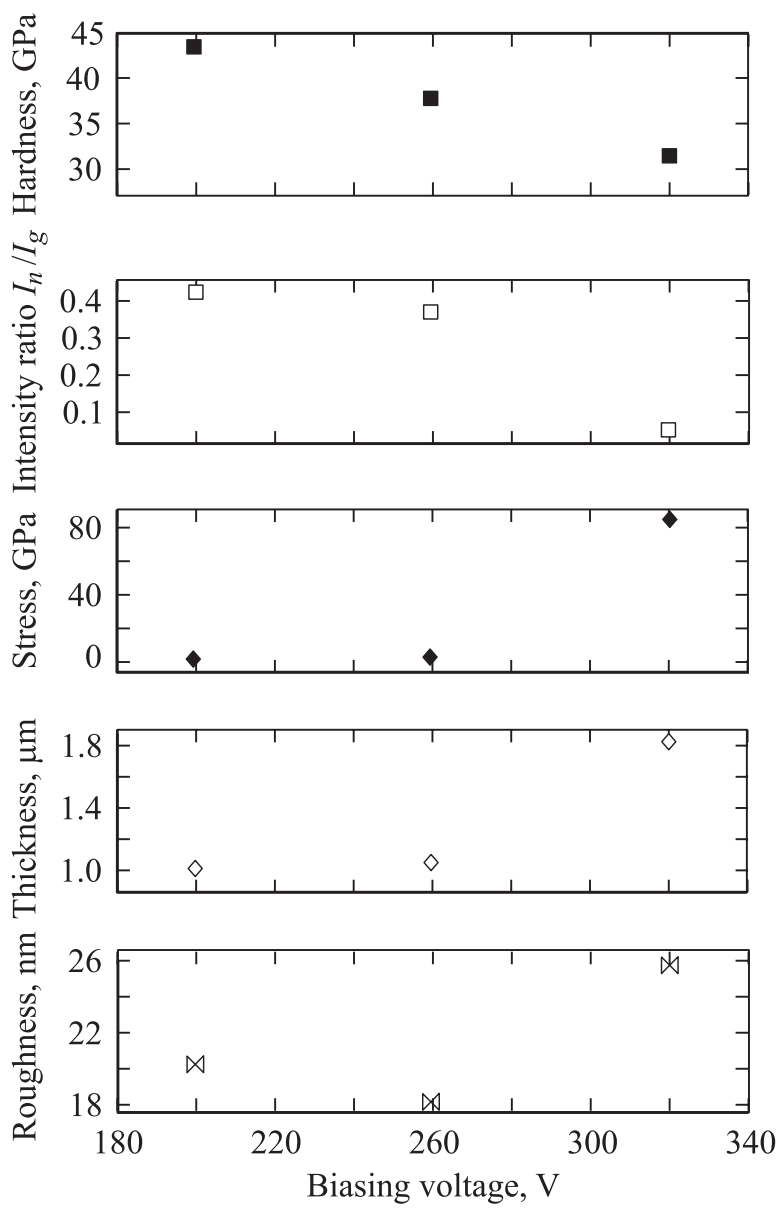

Figure 5. Plot of hardness, Raman intensity ratio of $\operatorname{NCD}\left(I_{n}\right)$ to graphitic $G$ band $\left(I_{g}\right)$, compressive stress, layer thickness, and rms surface roughness of the film, as a function of biasing voltage. 
observe such a large amount of stress. The strong adhesion in our films may be a result of subplantation of carbon ions with an optimized flux density into the substrate in the initial stages of growth. The layer thickness does not vary much while increasing the biasing voltage from 200 to $260 \mathrm{~V}$ but increases drastically at $320 \mathrm{~V}$, almost similarly as the stress in the films varied with the biasing voltage. The rms surface roughness of the films first decreases a little in the film grown at $260 \mathrm{~V}$ following a significant increase in the film grown at $320 \mathrm{~V}$.

It should be noticed here that, unlike the case of the growth of ta-C and DLC films by energetic carbon species [38-41], the hardness and stress in the films follow reverse trends with deposition parameters (except in a few cases in temperature series). For example, the hardness in the films decreases with increase in the biasing voltage and increases with increase in the methane concentration whereas the value of the stress in both series follows just the reverse trends. Therefore, interestingly, in our case the NCD film, grown at optimized condition for longer deposition time by the BEG process in the MPCVD system, shows the highest hardness with the lowest stress. Another advantage from this new route of growth is that any thickness can be grown because of having strong adhesion of the films to the substrate. The layer thickness first increases gradually up to $35 \mathrm{~min}$ of growth following a linear increase with time which is as expected. The calculated growth rate at optimized condition is as high as approximately $1 \mu \mathrm{m} / \mathrm{h}$.

\section{Conclusions}

Nanocristalline diamond films appear to be quite attractive as smooth diamond films. Several routes for the growth of chemical vapor deposited NCD films were reviewed. Some important results were also highlighted in the growth of NCD films on mirror polished Si substrates by a particular route termed as biased enhanced growth in the microwave plasma CVD system. In a special arrangement made in the microwave plasma CVD system, it was shown that the growth of NCD occurs while applying some certain negative voltage to the substrate. The NCD characteristics, as assessed by Raman spectroscopy and XRD, decreases, hardness decreases and stress in the films increases with increasing the bias voltage.

\section{References}

[1] D.G. Bhat, D.G. Johnson, A.P. Malshe, H. Naseem, W.D. Brown, L.W. Schaper, C.-H. Shen. Diamond Relat. Mater. 4, 921 (1995).

[2] A.K. Gangopadhyay, M.A. Tamor. Wear 169, 221 (1993).

[3] B. Bhusan. Diamond Relat. Mater. 8, 1985 (1999).

[4] A. Erdemir, G.R. Fenske, A.R. Krauss, D.M. Gruen, T. McCauley, R.T. Csencsits. Surf. Coat. Technol. 120-121, 565 (1999).

[5] S.K. Choi, D.Y. Yung, S.Y. Kweon, S.K. Jung. Thin Solid Films 279, 110 (1996).

[6] S. Hogmark, O. Hollman, A. Alahelisten, O. Hedenqvist. Wear 200, 225 (1996).
[7] D. Zhou, T.G. McCauley, L.C. Qin, A.R. Krauss, D.M. Gruen. J. Appl. Phys. 83, 540 (1998).

[8] T. Sharda, S. Bhattacharyya. In: Encyclopedia of Nanoscience and Nanotechnology / Ed. H.S. Nalwa. American Scientific Publ., California, USA (2003).

[9] L.S. Pan, D.R. Kania. Diamond: Electronic Properties and Application. Luwer Academ. Publ., London (1995).

[10] W. Banholzer. Surf. Coat. Technol. 53, 1 (1992).

[11] D.M. Gruen. Ann. Rev. Mater. Sci. 29, 211 (1999).

[12] D.M. Gruen, X. Pan, A.R. Krauss, S. Liu, J. Luo, C.M. Foster. J. Vac. Sci. Technol. A 12, 1491 (1994).

[13] T. Lin, Y. Yu, T.S. Wee, Z.X. Shen, K.P. Loh. Appl. Phys. Lett. 77, 2692 (2000).

[14] K. Wu, E.G. Wang, J. Chen, N.S. Xu. J. Vac. Sci. Technol. B 17, 1059 (1999).

[15] K. Wu, E.G. Wang, Z.X. Cao, Z.L. Wang, X. Jiang. J. Appl. Phys. 88, 2967 (2000).

[16] J. Lee, B. Hong, R. Messier, R.W. Collins. Appl. Phys. Lett. 69, 1716 (1996).

[17] J. Lee, R.W. Collins, R. Messier, Y.E. Strausser. Appl. Phys. Lett. 70, 1527 (1997).

[18] K. Teii, H. Ito, M. Hori, T. Takeo, T. Goto. J. Appl. Phys. 87, 4572 (2000).

[19] S. Yugo, T. Kanai, T. Kimura, T. Muto. Appl. Phys. Lett. 58, 1036 (1991).

[20] S.T. Lee, H.Y. Peng, X.T. Zhou, N. Wang, C.S. Lee, I. Bello, Y. Lifshits. Science 287, 104 (2000).

[21] T. Sharda, T. Soga, T. Jimbo, M. Umeno. Diamond Relat. Mater. 9, 1331 (2000).

[22] T. Sharda, M. Umeno, T. Soga, T. Jimbo. Appl. Phys. Lett. 77, 4304 (2000).

[23] T. Sharda, T. Soga, T. Jimbo, M. Umeno. Diamond Relat. Mater. 10, 1592 (2001).

[24] W.B. Yang, F.X. Lu, Z.X. Cao. J. Appl. Phys. 91, 10068 (2002).

[25] W. Zhu, G.P. Kochanski, S. Jin. Science 282, 1471 (1998).

[26] A. Gohl, A.N. Alimova, T. Habermann, A.L. Mescheryakova, D. Nau, G. Muller. J. Vac. Sci. Technol. B 17, 670 (1999).

[27] N.S. Xu, J. Chen, Y.T. Feng, M.J. McNallan. Nature 411, 283 (2001).

[28] E. Maillard-Schaller, O.M. Kuettel, L. Diederich, L. Schlapbach, V.V. Zhirnov, P.I. Belobrov. Diamond Relat. Mater. 8, 805 (1999).

[29] A. Hiraki. Appl. Surf. Sci. 162-163, 326 (2000).

[30] J. Robertson et al. Appl. Phys. Lett. 66, 3287 (1995).

[31] X. Jiang, C.-P. Klages, R. Zachai, M. Hartweg, H.-J. Fusser. Appl. Phys. Lett. 62, 3438 (1993).

[32] T. Sharda, T. Soga. To be published.

[33] A.C. Ferrari, J. Robertson. Phys. Rev. B 61, 14095 (2000).

[34] R.J. Nemanich, J.T. Glass, G. Lucovsky, R.E. Shorder. J. Vac. Sci. Technol. A 6, 1783 (1988).

[35] L.C. Nistor, J.V. Landuyt, V.G. Ralchenko, E.D. Obraztsova, A.A. Smolin. Diamond Relat. Mater. 6, 159 (1997).

[36] T. Sharda, M. Umeno, T. Soga, T. Jimbo. J. Appl. Phys. 89, 4874 (2001).

[37] F.C. Marques, R.G. Lacerda, G.Y. Odo, C.M. Lepienski. Thin Solid Films 332, 113 (1998).

[38] R.G. Lacerda, F.C. Marques. Appl. Phys. Lett. 73, 617 (1998).

[39] D.R. Mckenzie, D.A. Muller, B.A. Paithorpe. Phys. Rev. Lett. 67, 773 (1991).

[40] S. Sattel, J. Robertson, M. Scheib, H. Ehrhardt. Appl. Phys. Lett. 69, 497 (1996).

[41] S. Logothetidis, M. Gioti, P. Patsalas, C. Charitidis. Carbon 37, 765 (1999). 